\title{
Not so Typical: The Role of Epicardial Connections in Typical Flutter Refractory to Block across the Cavotricuspid Isthmus
}

\author{
Tharian Cherian ${ }^{1}$ and David Callans ${ }^{1}$ \\ ${ }^{1}$ University of Pennsylvania
}

June 15, 2020

There is emerging evidence that a keen understanding of atrial myofiber architecture is paramount to characterizing and treating atrial arrhythmias. Heterogeneity in the three dimensional anatomic structure of the atrium has previously been shown to create distinct endocardial and epicardial activation patterns during tachycardia in a canine model (1). In the clinical setting, the epicardial atrial architecture and its contribution to arrhythmias have been less well explored until recently. There has been a renewed interest in and appreciation of epicardial and interatrial connections, particularly in the treatment of left atrial arrhythmias refractory to traditional endocardial ablation.

The vein of Marshall has been postulated to harbor epicardial connections between the coronary sinus (CS) and the left atrium (LA), sustaining peri-mitral flutters refractory to endocardial ablation $(2,3)$. Conduction across the intercaval bundle, which connects the right atrium to the right superior pulmonary vein, has been reported to render isolation of the RSPV challenging requiring ablation at the carina or from the RA $(4,5)$. Similarly, the Bachmann bundle, the main pathway of interatrial connection, has been shown to be critical for maintenance of biatrial flutters $(6,7)$. More recently, conduction across the subepicardial septopulmonary bundle has been implicated in the maintenance roof dependent flutter despite isolation of the endocardial posterior wall (8). In contrast, the role of epicardial connections in sustaining right atrial arrhythmias has been less well described.

In this edition of the Journal, Chaumont et al. describe five patients who underwent electrophysiology study for typical atrial flutter, who had persistent arrhythmia despite achieving a line of block along the endocardial aspect of cavotricuspid isthmus (CTI) (9). Using entrainment and activation mapping during tachycardia, they identified atrial tissue critical to the arrhythmia circuit in the middle cardiac vein in four patients, and in close proximity to the CS ostium in one patient. Ablation at these locations restored sinus rhythm. Electroanatomic mapping was not available for most of these cases. Rather than a limitation, this absence allowed an amazing demonstration "old school" deductive electrophysiology.

The authors should be commended for this series of cases which demonstrate connections that sustain atrial flutter by bypassing the endocardially blocked CTI. This study elucidates the complex, layered physiology underpinning atrial flutter, considered among the simpler of arrhythmias we treat in the electrophysiology laboratory. The strength of the study is the elegant intracardiac electrograms for each case which allowed the authors to infer the mechanism of refractory arrhythmia and eliminate it by targeting critical areas guided by EGMs within the coronary venous system. Prior studies of atrial fibrillation have suggested that epicardialendocardial breakthrough maybe an important mechanism in maintenance of persistent AF (10). It appears that a similar mechanism maybe responsible for maintaining typical flutter refractory to endocardial CTI ablation.

Based on their findings, the authors propose a CS to low right atrium (RA) epicardial connection in the first four patients, and an RA to RA epicardial connection in one patient critical to the tachycardia circuit. 
Anatomically, however, it is unclear whether discrete connections akin to accessory pathways exist between these regions of interest to explain the observed findings. It is more likely that the atrial flutter circuit encompasses the entire thickness of the atrium, and owing to fiber orientation across the two layers, there are regions where the endocardial and epicardial surfaces communicate with each other. At these locations we appreciate the epicardial component of persistent flutter once the endocardium is ablated and line of block is achieved but tachycardia continues uninterrupted. This concept is illustrated in Figure 1, which demonstrates a case of persistent mitral annular flutter refractory to endocardial mitral annular line. Epicardial conduction necessary for maintaining tachycardia was observed after endocardial ablation, and ablation from the coronary sinus slowed and terminated the tachycardia.

The advent of high resolution 3-dimensional mapping systems has allowed characterization of atrial activation patterns in detail during tachycardia. Pathik et al investigated epicardial-endocardial breakthrough in activation mapping of right atrial macro-reentry tachycardia in 26 patients (11). They defined breakthrough as the presence of focal endocardial activation with radial spread unaccounted for by an endocardial wavefront, with same timing on every tachycardia cycle. Epicardial-endocardial breakthrough was observed in over $50 \%$ of the patients, with majority at the posterior RA, and one each at cavotricuspid isthmus postablation, RA septum, and the inferolateral RA. In four patients, areas of breakthrough were within the tachycardia circuit, and in one patient the breakthrough region was critical for arrhythmia maintenance. In all cases, breakthrough sites were adjacent to endocardial slowing or line of block - as mentioned above this finding is not entirely surprising, given that endocardial block is necessary to observe epicardial breakthrough while activation mapping.

A detailed morphologic and histologic study of the inferior right atrial isthmus by Cabrera et al may provide some anatomical insight to explain the current study findings (12). The authors establish the isthmus to be an anatomically heterogeneous region, with the anterior aspect being consistently muscular, while the posterior membranous and the middle trabeculated aspects having variable ratios of muscle fibers to fibrofatty tissue, with myocardial bundles extending from terminal crest toward the Eustachian ridge to cover the mouth of the coronary sinus. In refractory atrial flutter following endocardial CT ablation, it maybe that ablation from the CS allows the elimination of residual conduction through these muscle fibers which is critical for maintenance of tachycardia.

In conclusion, Chaumont et al should be congratulated for elegantly demonstrating the multi-layer physiological architecture of typical atrial flutter - a reflection of the anatomic complexity and heterogeneity of the cavotricuspid isthmus and its inputs, and of the atrial musculature in general. Appreciation of this complexity will undoubtedly empower us to characterize and treat this arrhythmia and others more effectively.

\section{References}

1. Schuessler RB, Kawamoto T, Hand DE, Mitsuno M, Bromberg BI, Cox JL, Boineau JP. Simultaneous epicardial and endocardial activation sequence mapping in the isolated canine right atrium. Circulation . $1993 ; 88: 250-263$

2. Briceño DF, Valderrábano M. Recurrent perimitral flutter due to vein of marshall epicardial connections bypassing the mitral isthmus: response to ethanol infusion. Circ Arrhythm Electrophysiol . 2014;7 :988-989.

3. Rodriguez-Manero M, Schurmann P, Valderrabano M. Ligament and vein of Marshall: a therapeutic opportunity in atrial fibrillation. Heart Rhythm . 2016;13 :593-601.

4. Patel PJ, D'Souza B, Saha P, Chik WW, Riley MP, Garcia FC. Electroanatomic mapping of the intercaval bundle in atrial fibrillation. Circ Arrhythm Electrophysiol . 2014;7 :1262-1267

5. Yoshida K, Baba M, Shinoda Y, et al. Epicardial connection between the right-sided pulmonary venous carina and right atrium in patients with atrial fibrillation: A possible mechanism for preclusion of pulmonary vein isolation without carina ablation. Heart Rhythm . 2019;16:671-678.

6. Kawamura I, Fukamizu S, Miyazawa S, Hojo R. Biatrial tachycardia utilizing Bachmann bundle. Heart 
Rhythm . 2018;15 :1277.

7. Gracia E, Fan R. Biatrial flutter circuit involving an anomalous insertion of the Bachmann bundle into the superior vena cava.HeartRhythm Case Rep . 2018;4 (8):353-355.

8. Garcia F, Enriquez A, Arroyo A, Supple G, Marchlinski F, Saenz L. Roof dependent atrial flutter with an epicardial component: Role of the Septopulmonary bundle. J Cardiovasc Electrophysiol.2019;30:1159-1163.

9. Chaumont C, Nadir S, Arnaud S, Latcu D, Anselme F. Electrophysiologic evidence of epicardial connections between low right atrium and remote right atrial region or coronary sinus musculature: relevance for catheter ablation of typical atrial flutter. J Cardiovasc Electrophysiol. 2020;

10. de Groot NM, Houben RP, Smeets JL, Boersma E, Schotten U, Schalij MJ, Crijns H, Allessie MA. Electropathological substrate of longstanding persistent atrial fibrillation in patients with structural heart disease: epicardial breakthrough. Circulation. 2010;122 : 1674-1682.

11. Pathik B, Lee G, Sacher F, Haissaguerre M, Jais P, Massoullie G, Derval N, Sanders P, Kistler P, Kalman J. Epicardial-endocardial breakthrough during stable atrial macroreentry: evidence from ultrahigh-resolution 3-dimensional mapping. HeartRhythm2017;14:1200-1207.

12. Cabrera JA, Sanchez-Quintana D, Farre J, Rubio JM, Ho SY. The inferior right atrial isthmus: further architectural insights for current and coming ablation technologies. J Cardiovasc Electrophysiol. 2005;16 :402-408

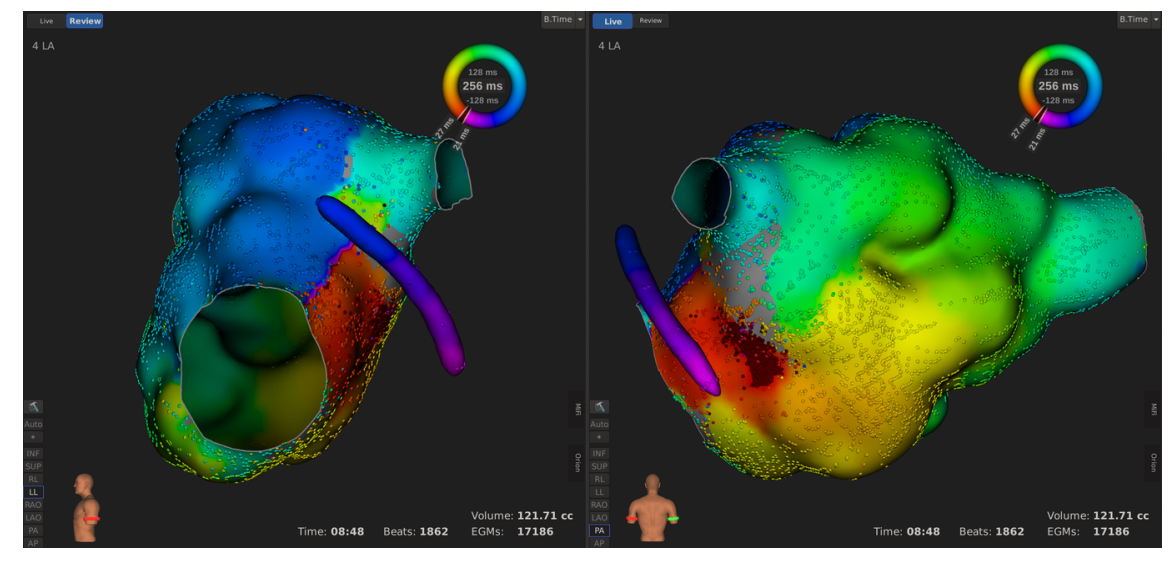

(a)

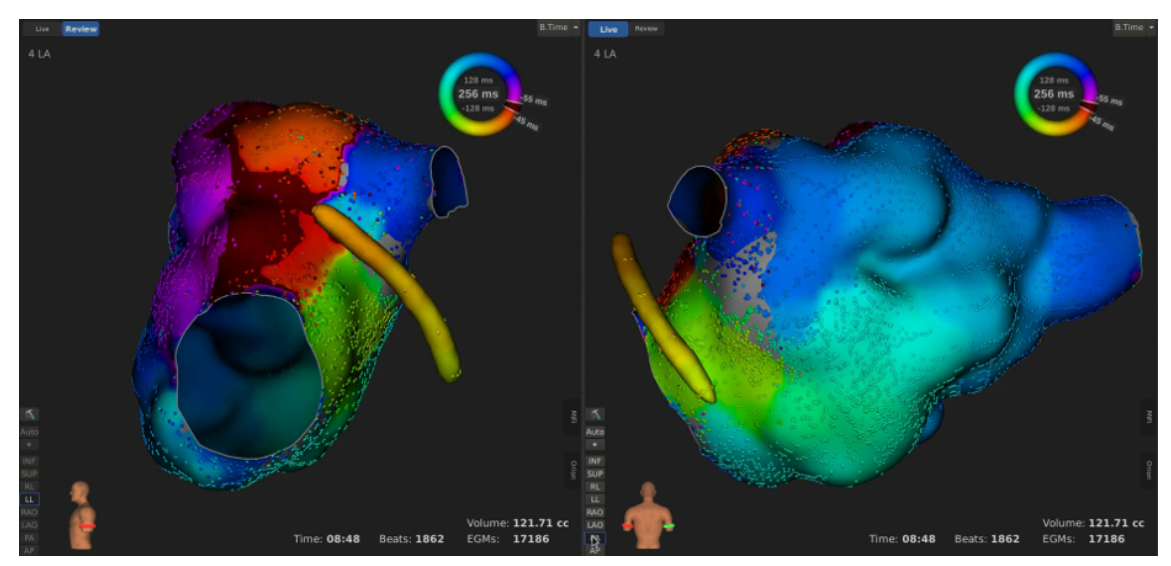




\section{(b)}

Figure 1. Activation mapping of the left atrium and coronary sinus in a patient who presented with clockwise mitral annular flutter. (a) Activation mapping prior to endocardial ablation showed clockwise mitral annular flutter with passive activation of the coronary sinus (CS). (b) Following endocardial ablation with creation of posterolateral mitral annular (MA) line, epicardial conduction involving the CS was demonstrated, which maintains tachycardia. Ablation from within the CS across the endocardial posterolateral MA line slowed and terminated the flutter, and bidirectional block across the line was confirmed. 